\title{
A polymer gel index-matched to water enables diverse applications in fluorescence microscopy
}
Xiaofei Han ${ }^{1,2^{*}, \wedge}$, Yijun Su${ }^{1,3^{*}, \wedge}$, Hamilton White ${ }^{4,5^{*}, \wedge}$, Kate M. O’Neill ${ }^{6,7}$, Nicole Y. Morgan ${ }^{8}$, Ryan Christensen ${ }^{1}$, Deepika Potarazu ${ }^{1}$, Harshad D. Vishwasrao ${ }^{3}$, Stephen $\mathrm{Xu}^{1}$, Yilun Sun ${ }^{9}$, Shar-yin Huang ${ }^{9}$, Mark W. Moyle ${ }^{10}$, Qionghai Dai ${ }^{2}$, Yves Pommier ${ }^{9}$, Edward Giniger ${ }^{6}$, Dirk R. Albrecht ${ }^{4,11}$, \&, Roland Probst ${ }^{12,}$, , Hari Shroff ${ }^{1,3,13, \&}$

1. Laboratory of High Resolution Optical Imaging, National Institute of Biomedical Imaging and Bioengineering, National Institutes of Health, Bethesda, Maryland, USA

2. Department of Automation, Tsinghua University, 100084, Beijing, China

3. Advanced Imaging and Microscopy Resource, National Institutes of Health, Bethesda, Maryland, USA

4. Department of Biomedical Engineering, Worcester Polytechnic Institute, 100 Institute Road, Worcester, MA, 01609, USA

5. Department of Neurobiology, University of Massachusetts Medical School, Worcester, MA 01655, USA

6. National Institute of Neurological Disorders and Stroke, NIH, Bethesda, MD, 20892, USA.

7. Institute for Physical Science and Technology, University of Maryland College Park, College Park, Maryland, USA

8. National Institute of Biomedical Imaging and Bioengineering, National Institutes of Health, Bethesda, Maryland, USA

9. Developmental Therapeutics Branch and Laboratory of Molecular Pharmacology, Center for Cancer Research, National Institutes of Health, Bethesda, Maryland, USA

10. Department of Neuroscience and Department of Cell Biology, Yale University School of Medicine, New Haven, CT 06536, USA.

11. Department of Biology and Biotechnology, Worcester Polytechnic Institute, 100 Institute Road, Worcester, MA, 01609, USA.

12. ACUITYnano, Innovation in Biomedical Imaging, North Bethesda, Maryland, USA

13. Marine Biological Laboratory Fellows Program, Woods Hole, MA, USA

*Equal contribution

\& Equal contribution

^ Correspondence to hxf16@mails.tsinghua.edu.cn, suy4nih@gmail.com, hawhite@wpi.edu

\section{Abstract}

We demonstrate diffraction-limited and super-resolution imaging through thick layers (tenshundreds of microns) of BIO-133, a biocompatible, UV-curable, commercially available polymer with a refractive index (RI) matched to water. We show that cells can be directly grown on BIO-

41133 substrates without the need for surface passivation and use this capability to perform

42 extended time-lapse volumetric imaging of cellular dynamics 1) at isotropic resolution using

43 dual-view light-sheet microscopy, and 2) at super-resolution using instant structured 
illumination microscopy. BIO-133 also enables immobilization of 1) Drosophila tissue, allowing us to track membrane puncta in pioneer neurons, and 2) Caenorhabditis elegans, which allows us to image and inspect fine neural structure and to track pan-neuronal calcium activity over hundreds of volumes. Finally, BIO-133 is compatible with other microfluidic materials, enabling optical and chemical perturbation of immobilized samples, as we demonstrate by performing drug and optogenetic stimulation on cells and C. elegans.

\section{Introduction}

Fluorescence microscopy spurs biological discovery, especially if imaging is performed at high spatiotemporal resolution and under physiologically relevant conditions. Coupling fluorescence microscopy with strategies for immobilizing or confining samples enables further applications, particularly when studying organisms that move rapidly. For example, the transparency and genetic accessibility ${ }^{1}$ of the nematode $C$. elegans has made it an ideal system for studying the growth, morphology and function of individual cells in the context of the whole organism ${ }^{2,3}$; yet imaging the living animal without motion blur usually requires immobilization with chemical ${ }^{4,5}$, steric ${ }^{6,7}$, or microfluidic ${ }^{8-11}$ means.

Microfluidic systems provide efficient immobilization and handling ${ }^{12-18}$ for studying cellular morphology ${ }^{19,20}$ and dynamics ${ }^{21}$, neuronal function ${ }^{22-24}$, behavior ${ }^{25-27}$, and lifespan ${ }^{28,29}$. Hydrogels (either independently7,30 or in conjunction with microfluidics ${ }^{31}$ ) have also been demonstrated as highly useful materials with tunable mechanical ${ }^{32}$, diffusive, and optical properties $^{33}$ that are well-suited for long-term imaging applications ${ }^{7,34,35}$.

Unfortunately, relatively few attempts have been made to index-match immobilization devices $^{36-39}$. The high refractive index $(R I, n)$ of materials commonly used in microfluidics, such as polydimethoxylsilane (PDMS), causes significant optical aberrations ${ }^{40,41}$ due to the RI mismatch that occurs at the interface between the polymer ( $n_{\text {PDMS }} \sim 1.41$ ) and an aqueous sample ( $n_{\text {water }}=1.33$ ). These aberrations severely degrade image focus, resolution, and signal, compromising the performance of immobilization devices by reducing the information content of the resulting data. Hydrogels offer a lower RI (n ranging from $1.34-1.41$ ) depending on thickness and polymerization conditions ${ }^{33}$. Although the RI of these materials is better matched to living samples, even a small mismatch in RI causes a noticeable deterioration in image quality ${ }^{42}$. Image degradation is particularly obvious when using water-dipping lenses designed for imaging living samples, such as those employed in high-resolution light-sheet fluorescence microscopy (LSFM) ${ }^{43-45}$.

Here we demonstrate a broadly applicable refractive-index-matched specimen mounting method that introduces negligible aberration when imaging living samples with highresolution light-sheet microscopy and super-resolution microscopy. We show its utility in combination with microfluidics, enabling applications in high-resolution, volumetric imaging of cells, Drosophila tissue, and C. elegans adults and larvae. Our method takes advantage of the commercially available UV curable optical polymer BIO-133 (MY Polymers Ltd.) that has a refractive index matched to water $(n=1.333)$, is non-fluorescent, and is non-toxic. We show that 1) BIO-133 provides a gas permeable, inert, and biocompatible scaffold on which to grow and image tissue culture cells, 2) enables rapid tissue or animal encapsulation, and 3) is compatible with other microfluidic mounting schemes and optical or chemical perturbations. 


\section{Results}

BIO-133 does not introduce additional optical aberrations

We assessed the optical properties of BIO-133 by using dual-view light-sheet microscopy (diSPIM ${ }^{43,46}$ ) to image $100 \mathrm{~nm}$ yellow-green beads placed under polymer layers of progressively increasing thickness (Fig. 1, Methods). In the most common diSPIM implementation, two identical 0.8 numerical aperture (NA) water-dipping objectives mounted above a planar substrate (usually a glass coverslip) alternately illuminate the sample with a light sheet and detect the resulting fluorescence. Since both illumination and detection planes are angled at $\sim 45$ degrees with respect to the glass coverslip, imaging through a polymer gel with surface parallel to the coverslip will introduce significant lateral (' $x$ ' direction, Fig. 1a) and axial (' $z$ ' direction, Fig. 1a) aberrations if the gel's refractive index differs from that of water.

As predicted, we observed this effect when imaging beads embedded under polymers with different RIs (Fig. 1b, c). Under no polymer, images of beads approximate the system point spread function, with measured lateral and axial full width at half maximum (FWHM) $395.9+/-$ $7.7 \mathrm{~nm}$ and $1527.9+/-119.5 \mathrm{~nm}$ ( $N=70$ beads), respectively. Imaging beads under PDMS caused severe aberrations (Fig. 1c), more than doubling the lateral FWHM under a $25 \mu \mathrm{m}$ layer $(816.8+/-24.9 \mathrm{~nm})$ with progressive deterioration under thicker polymer layers (Fig. 1b). We also observed aberrations (Fig. 1b, c) under poly(ethylene-glycol) diacrylate (PEG-DA) ${ }^{7}$ and fluorinated ethylene polymer (FEP) ${ }^{47}$, albeit to lesser extent as the refractive indices of these polymers are closer to water. By contrast, beads imaged under BIO-133 showed negligible visual aberrations or measurable degradation in image quality (Fig. 1b, c, Supplementary Table 1), even under a $150 \mu \mathrm{m}$ thick film, the largest thickness we tested (lateral FWHM 416.5 +/- 8.5 $\mathrm{nm})$. We attribute this result to the refractive index of BIO-133, which we measured to be 1.333. We also verified, under $488 \mathrm{~nm}, 561 \mathrm{~nm}$, and $637 \mathrm{~nm}$ illumination, that BIO-133 introduces negligible autofluorescence (Supplementary Fig. 1).

High-and super-resolution imaging of cells through a layer of $\mathrm{BIO}-133$

BIO-133 has permeability to oxygen about 2-3 times greater than PDMS and has water repellent properties (Personal Communication, Ehud Shchori, My Polymers, Ltd.). These material properties are advantageous for maintaining physiologically relevant cell culture conditions. Thus, we investigated whether BIO-133 could provide an inert and biocompatible scaffold for single cell imaging (Supplementary Table 2a). After a curing and leaching treatment (Methods), U2OS cells seeded on a $50 \mu \mathrm{m}$ layer of BIO-133 adhered and displayed similar morphology and growth rate to cells grown on glass coverslips (Fig. 2a, Supplementary Fig. 2). Similar results were obtained using HCT-116 (human colon carcinoma) cells that express endogenous topoisomerase I-GFP, and we also observed similar expression and localization of tagged proteins compared to cells cultured on glass coverslips (Supplementary Figs. 2, 3).

To demonstrate that transfected cells seeded directly on BIO-133 could be imaged at high spatiotemporal resolution, we created BIO-133 substrates on PDMS supports (Supplementary Fig. 4) and imaged cells expressing mEmerald-Tomm20, a fluorescent marker 
132 of the outer mitochondrial membrane, through a $50 \mu \mathrm{m}$ thick BIO-133 layer using diSPIM (Fig.

$133 \mathbf{2 b}, \mathbf{c})$. The jointly registered and deconvolved data acquired from two views displayed isotropic

134 spatial resolution (Fig. 2c, d), allowing us to clearly visualize individual mitochondria and their

135 dynamics (Supplementary Video 1), including mitochondrial fusion and fission (Fig. 2d). We

136 also used BIO-133 in conjunction with diSPIM to construct a simple gravity-driven flow

137 cytometry setup, obtaining clear images of DAPI stained nuclei as they flowed through the

138 chamber (Supplementary Fig. 5, 6, Supplementary Video 2).

Next, we sought to image subcellular targets at spatial resolution beyond the diffraction limit, so we turned to instant structured illumination microscopy (iSIM) ${ }^{48}$, which enables highspeed super-resolution imaging. We again seeded U2OS cells expressing mEmerald-Tomm20 on a $50 \mu \mathrm{m}$ BIO-133 film, this time imaging them with iSIM using a water dipping lens in an inverted geometry (Fig. 2e). Again, BIO-133 provided aberration-free imaging, enabling us to visualize the internal mitochondrial space absent Tomm20 (Fig. 2f, g, Supplementary Video 3). We also visualized LAMP-1-GFP-stained lysosome dynamics in wild type HCT-116 cells grown on another BIO-133 film of $50 \mu \mathrm{m}$ thickness (Supplementary Fig. 7, Supplementary Video 4). As a third example, we grew multiple layers of HCT-116 cells on BIO-133, and immunostained the cells for lamin A/C, Tomm20, and actin (Fig. 2h), obtaining clear images of these structures through the volume of the sample (Fig. 2i, Supplementary Video 5). We conclude that BIO-133 is compatible with multicolor, super-resolution imaging in live and fixed targets.

BIO-133 enables subcellular imaging, segmentation, and tracking within immobilized living tissue

In addition to monitoring the dynamics of organelles within single cells, we also immobilized and imaged multicellular structures in flies and worms at high spatiotemporal resolution, using diSPIM in conjunction with BIO-133 for sample immobilization (Fig. 3, Supplementary Table $\mathbf{2 b}$ ). The developing Drosophila wing has long been a model for axon growth and neuronal pathfinding and differentiation ${ }^{49,50}$. More recently, spinning disk confocal microscopy was used to dissect the role of cytoskeletal organization and dynamics in shaping the morphogenesis and growth of the TSM1 pioneer sensory neuron axon in explanted earlypupal wing imaginal discs ${ }^{51,52}$. In those experiments, phototoxicity and photobleaching limited imaging duration to $\sim 30$ volumes, with image volumes acquired every 3 minutes. Wings were sandwiched between two glass coverslips to immobilize the preparation and prevent it from moving during imaging, but this scheme introduces unacceptable aberrations if imaging with the less phototoxic diSPIM. Instead, we immobilized wings with a thin layer of BIO-133 (Fig. 3a, Supplementary Fig. 8), which enabled sustained volumetric imaging with diSPIM. We acquired 360 single-view volumes (5 s inter-volume interval, spanning 30 minutes) of tdTomato-CD4 expressed in TSM1 and the neighboring L3 neuron, marking neuronal membranes (Fig. $\mathbf{3 b}$ ). In addition to observing slower remodeling of the TSM1 growth cone (Fig. 3c, top) our imaging rate also enabled us to capture rapid movement of membrane-labeled puncta that appeared to traffic along the L3 axon shaft (Fig. 3c, bottom, Supplementary Video 6). Puncta were also

173 evident in comparative spinning disk confocal datasets (Supplementary Fig. 9). 
176 worms into the channels via suction (Fig. 3a), we imaged fine structures (Fig. 3d) and functional 177 activity (Fig. 3e-j) in living animals at isotropic resolution. Animals were sufficiently immobile 178 that we could serially acquire and fuse the two diSPIM views ${ }^{54}$ to obtain reconstructions free of 179 motion blur. In strains expressing GFP sparsely targeted to a few neurons, we resolved axons 180 and dendrites (likely from amphid neurons, Fig. 3d) within anesthetized C. elegans. When 181 imaging the genetically encoded calcium indicator GCaMP6s ${ }^{55}$ and mCherry targeted pan182 neuronally ${ }^{56}$ in immobilized adult animals without anesthetic, our volume imaging rate of 1.25 $183 \mathrm{~Hz}$ (simultaneous acquisition of red and green channels) enabled us to segment and track 126 184 nuclei in the animal head (Fig. 3e,f Supplementary Video 7), permitting inspection of 185 spontaneously active nuclei (Fig. 3f, g) over our 450 volume (6 minute) experiment. Intriguingly, we observed a pair of nuclei (\#79 and \#15, Fig. 3e, g) that exhibited in-phase, rhythmic activity with slow (45-80 s) period (Supplementary Video 7), as well as nuclei showing out-of-phase activity with respect to this pair (\#27). In another experiment, we simply embedded $C$. elegans larvae expressing the same pan-nuclear GCaMP6s marker in a cured disk of BIO-133 (Fig. 3a), recording volumes from one side to obtain volumes at $4 \mathrm{~Hz}$, for 250 volumes. Despite the poorer axial resolution of single-sided imaging, and the smaller size of the larval nuclei, we were able to again segment and track 110 nuclei in the head of the animal, identifying calcium transients in spontaneously active nuclei with a time resolution of $0.25 \mathrm{~s}$ (Fig. $\mathbf{3 h}$-j, Supplementary Video 8).

The droplet-based design also enabled easy recovery of animals post-imaging. 26 / 28 animals were recovered even $\sim 12$ hours after embedding, confirming our suspicion that cured BIO-133 is inert, gas permeable, water repellant, and does not obviously affect animal viability (the remaining two animals died within the BIO-133 capsule due to internal hatching of embryos within the animals). The water repellency of BIO-133 likely contributes to retaining the animal's intrinsic hydration and thus viability during encapsulation. The ease at which $C$. elegans can be immobilized and imaged at high spatiotemporal resolution suggests useful synergy with multicolor strategies that permit unambiguous neural identification ${ }^{23}$.

\section{BIO-133 is compatible with chemical and optogenetic perturbations}

The ability to specifically perturb and subsequently follow biological processes by observing morphological or functional changes is valuable in dissecting biological processes. We conducted several studies to show that BIO-133-mounted samples are compatible with such perturbations (Fig. 4). First, we conducted a simple drug assay by modifying our BIO133/PDMS cellular scaffolds (Fig. 2b, Supplementary Fig. 11) so that U2OS cells could be exposed (Fig. 4a) to carbonyl cyanide m-chlorophenyl hydrazine (CCCP), an inhibitor of oxidative phosphorylation. Because we could clearly observe cells through the BIO-133 layer using diSPIM, we observed that, compared to control cells in a neighboring well (Fig. 4a-c), within minutes of exposure the treated cells showed mitochondrial fragmentation, eventually exhibiting major disruption to the mitochondrial network (Fig. 4b, c, Supplementary Video 9).

Chemically stimulating animals directly embedded in BIO-133 is difficult, since BIO-133 is not permeable to aqueous solutions. One solution is to introduce chemicals via microfluidic channels (such as those shown in Fig. 3d-g). Alternatively, we explored using PEG-DA for immobilization and aqueous permeability, above a microfluidic layer for stimulus introduction 
220 and control, and beneath a BIO-133 layer to enclose the fluidic path. Using a thin PEG-DA disk

221 allows easy transfer of different embedded organisms on the same imaging setup, and

222 repeated imaging of the same animals over many hours if desired. We constructed a hybrid

223 multi-material device composed of a PDMS microfluidic base bonded to a BIO-133 upper membrane that sealed in a small PEG-DA disk containing tens of embedded nematodes ${ }^{7}$ (Supplementary Fig. 12, Fig. 4a). Chemicals applied via flow channels diffuse into the PEG-DA disk, evoking neural responses that can be imaged through the BIO-133 viewing layer with widefield microscopy or diSPIM (Fig. 4d-g, Methods). We embedded 15 animals expressing GCaMP2.2b in AWA chemosensory neurons ${ }^{57}$ in a PEG-DA disk and applied $1.1 \mu \mathrm{M}$ diacetyl pulses, which directly activate these neurons via the ODR-10 chemoreceptor ${ }^{57,58}$. Using widefield microscopy, we recorded robust calcium transients from all animals and observed a characteristic sensory adaptation to repeated stimulation (Fig. 4e). The initiation of sensory neural responses varied slightly due to diffusion of diacetyl stimulus through the PEG-DA disk to animals embedded in different planes (Supplementary Fig. 13). Next, we examined individual neuron responses by using the same apparatus with diSPIM (Fig. 4f,g Supplementary Video 10). Our imaging provided sufficient spatial resolution to distinguish subcellular responses, observing faster on and off dynamics of fluorescent transients in the dendrites than in the 237 soma 59. directly transmissible through BIO-133, and these can be applied directly to cells and organisms embedded in the polymer in a simpler preparation. Optogenetic neural activation is a particularly advantageous tool, allowing remote light-induced activation or suppression of neurons. We embedded nematodes expressing the red light activated cation channel

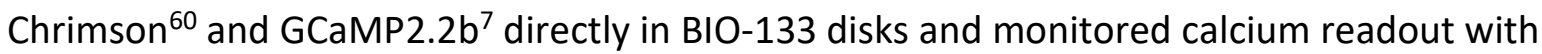
diSPIM during repeated red light stimulation pulses (Fig. 4a,h,i, Supplementary Video 11). We observed increases in fluorescence after each stimulus (Fig. 4h), and again could clearly localize such transients to subcellular areas including soma, dendrite, and axon (Fig. 4i).

\section{Discussion}

BIO-133 is commercially available, rapidly curing, gas permeable, inert, water repellent, and biocompatible. It is not autofluorescent under visible illumination and does not introduce additional aberration when imaging with water-dipping or water-immersion objective lenses designed for aqueous specimens. These characteristics make it well-suited to microfluidic experiments under physiological conditions, particularly with the many LSFM systems that use such lenses. We suspect that capillary mounting ${ }^{47}$, often used for mounting zebrafish in LSFM, could be improved if $\mathrm{BIO}-133$ were used instead of the FEP material commonly used in this application. Our finding that cells can be directly grown on BIO-133 without additional surface treatment may prove useful in non-standard LSFM geometries that previously employed RImatched materials with passivated surfaces ${ }^{45}$, or in ultra-high-throughput light-sheet imaging ${ }^{61,62}$.

261 We also found that BIO-133 does not noticeably degrade imaging performed in more

262 traditional inverted microscope geometries, including in super-resolution imaging. This

263 capability suggests that using BIO-133 could improve imaging in studies of cell morphology, 
mechanics, migration, and motility, e.g., when using micropillars ${ }^{20,63}$ or in traction force microscopy ${ }^{64}$.

We bonded BIO-133 to glass and PDMS with silicone-based adhesive tape, or reversibly to glass via van der Waals forces. The elastic modulus of cured BIO-133 (5 MPa) is similar to that of PDMS (3.7 MPa). Thus, similar to PDMS ${ }^{65}$, BIO-133 conforms to minor imperfections in glass and bonds to it by weak van der Waal forces, creating a reversible bond and a watertight seal. We suspect further tuning of adhesive, optical and mechanical properties of this intriguing polymer is possible but will depend on knowing the chemical formula, which is currently proprietary.

\section{Author Contributions}

Conceived project: R.P., H.S. Designed experiments: X.H., Y.S., H.W., K.M.O., H.D.V., E.G., D.A., R.P., H.S. Provided technical advice and resources for microfluidics: N.M., R.P., H.W., D.A. Provided biological advice: R.C., Y.S., S.H., Y.P. Created new reagents: S.H., Y.P, M.W.M. Performed experiments: X.H., Y.S., H.W., K.M.O., D.P., H.D.V., R.P. Tracked nuclei in GCaMP imaging experiments: X.H., Y.S., S.X. Wrote paper with input from all authors: X.H., Y.S., H.W., D.A., R.P., H.S. Supervised research: Q.D., Y.P., E.G., D.A., R.P., H.S. Directed research: H.S.

\section{Acknowledgements}

This research was funded in part by the National Institute of Biomedical Imaging and Bioengineering, the National Institute of Neurological Disorders and Stroke, and the Center for Cancer Research of the National Cancer Institute within the National Institutes of Health (Z01 BC 006161), and the National Science Foundation (CBET 1605679). We thank George Patterson for the use of his cell culture facilities, Leighton Duncan and Daniel Colón-Ramos for kindly providing strains and for conducting initial pilot experiments and their careful read, Evan Ardiel and Eviatar Yemini for providing helpful feedback on pan-nuclear GCaMP recordings, Ron Zohar and Ehud Shchori for providing useful information on BIO-133, and Hank Eden for providing helpful feedback on the manuscript. K.M.O. and E.G. were supported by NINDS Z01-NS003013 to E.G. K.M.O. was also jointly supported by AFOSR grant number FA9550-16-1-0052 to W. Losert at UMD College Park. M.W.M was supported by NIH grant F32-NS098616.

Disclaimer: The NIH, its officers, and staff do not recommend or endorse any company, product, or service.

\section{Methods}

\section{Sample preparation}

U2OS, wild type (WT) HCT-116, and HCT-116 TOP1-GFP cell culture U2OS (ATCC, HTB-96), WT HCT-116 (ATCC, CCL-247), and HCT-116 TOP1-GFP (see below) cells $\mathrm{CO}_{2}$. 
To tag the genomic topoisomerase I (TOP1) in WT HCT-116 cells, sequence

CCTCACTTGCCCTCGTGCCT targeting a CRISPR site 77nt after the stop codon of TOP1 was cloned into pX330. Homology arms (of $1 \mathrm{~kb}$ ) upstream and downstream of the target site were cloned to flank a blasticidin - resistance gene, where the upstream homology arm was modified to replace the stop codon with a GFP domain connected to the protein-coding region of the last exon of TOP1 via a short poly-lysine linker. Both constructs were co-transfected into WTHCT116 cell, followed by selection with $5 \mu \mathrm{g} / \mathrm{mL}$ of blasticidin 48 hours post transfection. GFPpositive cells were further selected by FACS. Reagent (Sigma, 6366236001). The transfection mixture contained $100 \mu \mathrm{L} 1 \mathrm{X}$ PBS, $2 \mu \mathrm{L}$ Transfection Reagent, and 200-1000 ng plasmid DNA. Cells were imaged 24-48 hours after

322 transfection.

\section{C. elegans samples}

Nematode strains were grown on NGM plates seeded with OP50 bacteria. C. elegans imaged as young adults were synchronized by picking $L 4$ stage worms 24 hours prior to the experiment and transferring them to seeded plates, and C. elegans imaged as larvae were directly picked from plates. Strain DCR6268 (olaEx3632[pttx-3b::SL2::Pleckstrin homology domain::GFP::unc-54 3'UTR + pelt-7::mCh::NLS::unc-54 3'UTR]) was used for imaging axons and dendrites (Fig. 3d). olaEx3632 was made by injecting plasmid DACR2285 (pttx-3b::SL2::Pleckstrin homology domain::GFP::unc-54 3'UTR) at $25 \mathrm{ng} / \mathrm{uL}$ and DACR2436 (pelt-7::mCh::NLS::unc-54 3'UTR) at 10 ng/ $\mu$ L. Strain AML32 ${ }^{56}$ (wtfls5 [rab-3p::NLS::GCaMP6s + rab-3p::NLS::tagRFP]) was used for pannuclear neuronal calcium imaging (Fig. 3e-j); strain NZ1091(kyls587 [gpa-6p::GCaMP2.2b; unc122p::dsRed]; kyls5662 [odr-7p::Chrimson:SL2:mCherry; elt-2p::mCherry] ${ }^{7}$ ) was used for chemical (Fig. 4d-g) and optogenetic stimulation (Fig. 4h, i) . For optogenetic stimulation, L4 stage animals were transferred to agar plates seeded with $62.5 \mu \mathrm{M}$ all trans-retinal (ATR, Sigma Aldrich, R2500) for over 12 hours.

\section{Drosophila samples}

Drosophila stocks were obtained from the Bloomington Drosophila Stock Center: neur-GAL4 (BL6393) and UAS-CD4-td-Tomato (BL35837). White prepupae were selected and aged for 13$14 \mathrm{~h}$ at $18^{\circ} \mathrm{C}$ followed by $1 \mathrm{~h}$ at $25^{\circ} \mathrm{C}$ (equivalent to $7.5-8 \mathrm{~h}$ at $25^{\circ} \mathrm{C}$ ). These aged pupae were dissected in fresh culture media (CM; Schneider's Drosophila media $+10 \%$ fetal bovine serum, both from Life Technologies), and wing discs were isolated from the aged pupae and stored in fresh CM prior to mounting with BIO-133.

\section{Characterizing the optical properties of polymers}

Characterization of aberrations by visualizing fluorescent beads under different polymer layers 
$100 \mathrm{~nm}$ diameter yellow-green fluorescent beads (Invitrogen, F8803, 1:10000 dilution in water) were coated on \#1.5 coverslips ( $24 \mathrm{~mm} \times 50 \mathrm{~mm}$, VWR, 48393-241) coated with $0.1 \% \mathrm{w} / \mathrm{v}$ polyL-lysine (Sigma, P8920-100ML). We placed spacers (Precision Brand, 44910) of variable thickness on one of the coverslips and deposited droplets of BIO-133, 10\% PEG-DA (ESIBIO, GS705) or UV-curable PDMS (Shin-Etsu Chemical, KER-4690) on the beads. The droplet was then covered with another coverslip coated with beads and compressed with an iron ring. BIO133 and PEG-DA hydrogels were crosslinked at $312 \mathrm{~nm}$ (Spectroline ENB-280C) for 2 minutes. PDMS was crosslinked at $312 \mathrm{~nm}$ for 5 minutes and post-cured at room temperature for one day. Once cured, we separated the two cover glasses and kept the one with polymer/hydrogel on it. For imaging through FEP films of different thickness (CS Hyde, 23-1FEP-24, $25 \mu \mathrm{m}$; 232FEP-24, $50 \mu \mathrm{m}$; 23-3FEP-24, $75 \mu \mathrm{m}$; 23-5FEP-24, $125 \mu \mathrm{m}$ ), we immersed beads in $1 \mu \mathrm{L}$ water and covered the sample with the FEP film. All bead images were acquired with a symmetric 0.8/0.8 NA diSPIM ${ }^{43}$.

Measurement of refractive index of polymers A refractometer (American Optical) was used to measure the refractive index of pure water, BIO-133 film (My Polymer, BIO-133, $25 \mu \mathrm{m}$ ), PDMS film (Shin-Etsu Chemical, KER-4690, $25 \mu \mathrm{m}$ ), FEP film (CS Hyde, 23-1FEP-24, $25 \mu \mathrm{m}$ ), and 10\% PEG-DA hydrogel (100 $\mu \mathrm{m})$. The refractive index for each material was measured 3 times and the average value reported in Fig. 1.

\section{Measurement of BIO-133 autofluorescence}

A $50 \mu \mathrm{m}$ thick BIO-133 film was deposited on a glass bottom dish (MatTek, P35G-1.5-14-C). Images were acquired both on the BIO-133 area and an area without BIO-133, using an instant structured illumination microscope (iSIM ${ }^{48}$ ) with $40 \mathrm{~ms}$ exposure time and $45 \mathrm{~mW} 488 \mathrm{~nm}$ excitation, $70 \mathrm{~mW} 561 \mathrm{~nm}$ excitation, or $90 \mathrm{~mW} 639 \mathrm{~nm}$ excitation (measured with a power meter immediately prior to the objective). Care was taken to ensure the illumination was focused within the BIO-133 film. The two images were subtracted to measure the autofluorescence of BIO-133 relative to glass (Supplementary Fig. 1).

\section{Cell growth and imaging using BIO-133 substrates}

Fabrication of BIO-133 cell culture wells for diSPIM experiments was positioned on a \#1.5 glass coverslip ( $24 \mathrm{~mm} \times 50 \mathrm{~mm}$, VWR 48393-241) between two 50 $\mu \mathrm{m}$ plastic spacers (Precision Brand, 44910), covered with another coverslip , compressed with a glass slide (Ted Pella, 260386) and cured with a UV lamp (365nm, Spectroline ENB-280C) for 15 minutes. After curing, the BIO-133 film was peeled off and exposed to UV light for another 2 hours in 70\% ethanol. To make the PDMS well, $15 \mathrm{~mL}$ PDMS (Dow Inc. Sylgard 184) was poured into a $10 \mathrm{~cm}$ plastic dish (Kord-Valmark, 2910) and cured for 2 hours at $80^{\circ} \mathrm{C}$ to obtain a $2 \mathrm{~mm}$ thick PDMS slab. We punched $2.5 \mathrm{~mm}$ diameter matching holes on the PDMS slab and a piece of double-sided tape (Adhesives Research, ARCare 90880) using a $2.5 \mathrm{~mm}$ diameter circular punch (Acuderm Inc., P2550). The PDMS slab and the tape were then cut into smaller pieces ( $\sim 5$ 
$\mathrm{mm}$ on a side) with a razor blade (Sparco, 01485). BIO-133 membranes, PDMS chunks and double-sided tape were further disinfected in $70 \%$ ethanol for 2 hours. After disinfection, the BIO-133 membrane was adhered to PDMS using the adhesive tape, so that the matching holes became wells for cell culture. After seeding and growing cells in wells, the assembly was flipped over for diSPIM imaging.

\section{Quantification of cell growth} Cured and leached $50 \mu \mathrm{m}$ thick BIO-133 films were deposited on glass bottom dishes (MatTek, P35G-1.5-14-C). Similar aliquots of U2OS (or HCT 116 TOP1-GFP) cells were seeded onto BIO133 films or on another glass bottom dish without BIO-133 (MatTek, P35G-1.5-14-C). Dishes seeded with cells were maintained between imaging experiments in an incubator at $37^{\circ} \mathrm{C}, 5 \%$ $\mathrm{CO}_{2}$. On each dish, a small area was selected and imaged using a widefield microscope equipped with a 10x/0.25 NA objective lens each day, for three days. Cells numbers were estimated from images with the Cell Counter ImageJ plugin (https://imagej.nih.gov/ij/plugins/cellcounter.html). Each experiment was repeated three times. Raw images were divided by Gaussian-blurred versions of themselves (sigma $=5$ pixels) to flat-field images prior to display (Fig. 2a, Supplementary Fig. 2).

Live cell imaging through BIO-133 with diSPIM (Addgene, 54281) directly on the BIO-133 bottomed well plate. The well plate was inverted and immersed in live cell imaging solution (Invitrogen, A14291DJ). Cells were imaged with a symmetric 0.8/0.8 NA diSPIM, through the BIO-133 layer. 50 volumes were acquired with $3 \mathrm{~s}$ intervals between dual-view volumes. Dual-view data were jointly deconvolved with ImageJ plugin DiSPIM Fusion ${ }^{54}$, and were drift- (with ImageJ plugin Correct 3D Drift (https://imagej.net/Correct_3D_Drift) and bleach- corrected (with ImageJ function Bleach Correction (https://imagej.net/Bleach Correction, exponential fitting method) prior to display. EGFP (Taraska Lab, NHLBI) on a $50 \mu \mathrm{m}$ thick BIO-133 film. The BIO-133 film was cured on a glass bottom dish (MatTek, P35G-1.5-14-C). A 60X, NA = 1.2 water objective (Olympus, PSF grade), correction collar adjusted to 0.17 , was used to image the cells through the glass and BIO-133 film using our home built iSIM system ${ }^{48}$ and $488 \mathrm{~nm}$ excitation. Volumes were acquired every 3 $\mathrm{s}$ for U2OS cells expressing mEmerald-Tomm 20 and every $7 \mathrm{~s}$ for WT HCT-116 cells expressing LAMP1-EGFP. We used an exposure time of $80 \mathrm{~ms}$, and a z-step of $0.25 \mu \mathrm{m}$ for U2OS cells and $0.5 \mu \mathrm{m}$ for WT HCT-116 cells. Live HCT-116 TOP1-GFP cultured on a $50 \mu \mathrm{m}$ thick BIO-133 film or on a glass bottom dish were imaged to acquire volumes with a step size of $0.5 \mu \mathrm{m}$. Raw images were deconvolved with the Richardson-Lucy algorithm for 20 iterations, destriped in Fourier space to remove striping artifacts ${ }^{66}$, and bleach corrected (https://imagej.net/Bleach Correction). A median filter with kernel size 0.5 pixel was applied to denoise mEmerald-Tomm20 and GFP-LAMP1 images prior to display. 
438 WT HCT-116 cells were cultured on a $50 \mu \mathrm{m}$ thick BIO-133 film on a glass bottom dish until a thick layer was visible by eye. Cells were fixed with 4\% paraformaldehyde (Electron Microscopy Sciences) in $1 \mathrm{X}$ PBS for 30 minutes at room temperature (RT). Cells were rinsed 3 times in $1 \mathrm{X}$ PBS and permeabilized with 0.1\% Triton X-100/PBS (Sigma, 93443) for 15 min at RT. Permeabilized cells were rinsed 3 times with 1X PBS and incubated in 1X PBS with primary antibody Rabbit- $\alpha$-Tomm20 (Abcam, ab186735) and Mouse- $\alpha$-LaminA/C (Abcam, ab244577) at a concentration of 1:100 for 1 hour at RT. After primary antibody staining, cells were washed in 1X PBS for $5 \mathrm{~min}$, three times. Cells were stained in 1X PBS with secondary antibody Donkey- $\alpha-$ Rabbit Alexa Fluor 488 (Jackson Immuno Research, 711-547-003), Donkey- $\alpha$-Mouse JF549 (Novusbio, NBP1-75119JF549) and Alexa Fluor 647 Phalloidin (Thermofisher, A22287) at a concentration of 1:100 for 1 hour at RT. Cells were washed in 0.1\% Triton X-100/PBS for 5 min, three times. In each spectral channel, 46 slices were acquired on iSIM with an exposure time of $100 \mathrm{~ms}$ and a z-step of $0.5 \mu \mathrm{m}$. Raw images were deconvolved with the Richardson-Lucy algorithm for 20 iterations and destriped in Fourier space to remove striping artifacts ${ }^{66}$. The 633 $\mathrm{nm}$ channel (Alexa Fluor 647 Phalloidin) was bleach corrected (https://imagej.net/Bleach Correction) across the $z$ stack to compensate for decreased signal further into the stack.

\section{Flow cytometry preparation}

Sample handling channels, $1 \mathrm{~mm}$ wide and $70 \mu \mathrm{m}$ high, were formed by pouring $20 \mathrm{~mL}$ PDMS (Dow Corning, Sylgard 184) on a positive mold made of packaging tape (Duck Brand) cut to the desired dimensions with a craft cutter (Silhouette Cameo) and stuck in a $10 \mathrm{~cm}$ Petri dish. A thin PDMS membrane ( $0.5 \mathrm{~mm}$ ) was air plasma (Harrick Plasma, PDC-32G (115V)) bonded to the channel surface. Holes at the endpoints of the channel were created by punching the PDMS membrane with a $1 \mathrm{~mm}$ diameter circular punch (Acuderm Inc., P150) after plasma treatment. A $400 \mu \mathrm{m}$ wide (142 $\mu \mathrm{m}$ height) imaging channel was cut directly from double-sided siliconbased adhesive tape (Adhesives Research, ARCare 90880) with a craft cutter and stuck to the PDMS device, thus creating a connection between the two sample handling channels in the lower layer. A thin BIO-133 membrane $(50 \mu \mathrm{m})$ was placed on top of the tape to seal the channel. Two thick PDMS pieces with holes ( $6 \mathrm{~mm}$ and $2 \mathrm{~mm}$ diameter) were cut and air plasma bonded to the device to provide fluidic access. When imaging, cells were added to the $6 \mathrm{~mm}$ diameter reservoir, and output tubing (Dow Corning, 508-004) was connected to the $2 \mathrm{~mm}$ hole. Flow speed was adjusted by changing the height of the output tubing. See also

\section{Supplementary Fig. 5.}

Flow cytometry imaging of fixed, DAPI-stained U2OS cells imaging with diSPIM. Fixed cells were added to the input port, producing steady flow through the channel after several minutes. 1000 frames of the same image plane were acquired with diSPIM at 50 frames per second under 'fixed sheet mode'.

\section{Live animal/tissue imaging through BIO-133 with diSPIM}


Live imaging of $\mathrm{BIO}-133$ embedded Drosophila wings

A $13 \mu \mathrm{m}$ thick BIO-133 film was created and cut into two rectangular pieces $(4.5 \mathrm{~mm} \times 10 \mathrm{~mm})$ and a square piece $(10 \mathrm{~mm} \times 10 \mathrm{~mm})$. The rectangular pieces were deposited on a $10 \mathrm{~cm}$ petri dish to form a $1 \mathrm{~mm}$ wide open-top channel. Early pupal fly wings were deposited into the channel (convex side up) with 20-40 $\mu \mathrm{L}$ culture media, the square BIO-133 piece placed on top to close the channel and additional culture media was carefully added to the dish (Fig. 3a, Supplementary Fig. 8). Single-view diSPIM imaging was then performed. 360 volumes were acquired with $5 \mathrm{~s}$ inter-volume spacing, over 30 minutes. Volumes were deconvolved with MATLAB and bleach corrected with ImageJ (https://imagej.net/Bleach Correction, exponential fitting method). Standard soft lithography techniques ${ }^{67}$ were used to fabricate an SU-8 (Kayaku Advanced Materials, formerly Microchem Corp.) master mold for sets of four microfluidic funnels for worm confinement as described ${ }^{53}$. To fabricate devices in BIO-133 (MY Polymers Ltd.), we placed two spacers (100 $\mu \mathrm{m}$, Precision Brand 44910) beside the pattern, poured polymer onto the mold, covered the mold with a glass slide and cured the polymer under a UV lamp $(365 \mathrm{~nm}$, Spectroline ENB-280C) for 2 minutes. After curing, we peeled the BIO-133 off the mold, punched inlet and outlet holes with a $1 \mathrm{~mm}$ diameter circular punch (Acuderm Inc., P150), and sealed the device to a \#1.5 cover glass (24 mm x $50 \mathrm{~mm}$, VWR 48393-241) with double-sided silicone-based adhesive tape (Adhesives Research, ARCare 90880). We cut out an aperture from a $10 \mathrm{~cm}$ petri dish and used UV-curable optical cement (Norland Products Inc., Norland Optical Adhesive NOA 68) to secure the coverslip carrying the microfluidic device over the aperture in the petri dish. Inlet and outlet tubing (Dow Corning, 508-004) was clamped to the assembly using a pair of hollow magnets (K\&J Magnetics, R211-N52) placed above and below the coverslip, as described ${ }^{68}$. Optical cement was again used to secure tubing to the magnets. See

\section{also Supplementary Fig. 10.}

Live imaging of $C$. elegans through $\mathrm{BIO}-133$ chambers

511 To load worms into the immobilization device, we added a drop of M9 buffer containing worms

512 to the inlet and created vacuum at the outlet using a syringe. Within several minutes (for a 4-

513 channel chip), worms were observed to align in the channels. The petri dish was then filled with 514 water and worms were imaged with symmetric 0.8/0.8 NA diSPIM. For structural imaging, we 515 added $0.25 \mathrm{mM}$ levamisole to the buffer to stop residual worm motion. For calcium imaging, 516 the outlet was connected to a peristaltic pump (Dolomite Microfluidics, 3200243) which 517 provided negative pressure to immobilize worms without using anesthetics. We simultaneously 518 imaged nuclei structure (TagRFP) and the nuclear-localized calcium response (GCaMP) with 488 $519 \mathrm{~nm}$ and $561 \mathrm{~nm}$ excitation (Coherent) and image splitting devices on the detection side 520 (Hamamatsu W-VIEW GEMINI), using a previously described fiber-coupled diSPIM system ${ }^{46}$. 521 Dual-view stacks were acquired every 0.8 s over 500 time points. Dual-color, dual-view images 522 were deconvolved and registered with ImageJ plugin DiSPIM Fusion ${ }^{54}$. 
525 C. elegans were directly transferred from agar plates into a drop ( $10 \mu \mathrm{L}$ ) of BIO-133 or $10 \%$ PEG-DA (ESIBIO GS705) on a \#1.5 cover glass ( $24 \mathrm{~mm} \times 50 \mathrm{~mm}, \mathrm{VWR}, 48393-241)$. The droplet was positioned between two $100 \mu \mathrm{m}$ spacers (Precision Brand, 44910), and was compressed by a glass slide followed by 2 -minute polymerization under a UV lamp (365nm, Spectroline ENB280C). After polymerization, worms were immobilized in the resulting gel disk. The gel disk was then placed in a $10 \mathrm{~cm}$ petri dish or a standard chamber for diSPIM imaging. Single-view stacks were acquired every $0.25 \mathrm{~s}$ for 250 time points. After imaging, worms could be released and checked for viability by gently breaking the droplet with forceps. In some experiments, we immersed the disks in $\mathrm{M} 9$ buffer for up to 12 hours, finding that live worms could also be recovered after this period.

\section{Tracking nuclei, calcium imaging analysis} TagRFP volumes were imported into Imaris and neurons tracked with Imaris for Tracking (https://imaris.oxinst.com/products/imaris-for-tracking) to obtain the center of each neuron at every timepoint. A custom MATLAB script was used to extract the calcium signal. For every neuron, the average intensity of TagRFP channel $I_{561}$ and the intensity of GCaMP channel $I_{488}$ were computed by averaging pixels within a $2 \mu \mathrm{m}$ (adult) or $1.5 \mu \mathrm{m}$ (larval) diameter sphere placed around each center position. $I_{561}$ and $I_{488}$ were calculated from dual-view deconvolved images (Fig. $3 \mathrm{f}, \mathrm{g}$ ) or single-view raw data (Fig. $3 \mathrm{i}, \mathrm{j}$ ). The ratio $\mathrm{R}=\mathrm{I}_{488} / \mathrm{I}_{561}$ was used to minimize non-GCaMP fluctuations. Neuronal activity for the datasets in Fig. 3 was reported as $d R / R=(R-$ $\left.R_{0}\right) / R_{0}$, where $R_{0}$ is the baseline for an individual neuron defined as its lower 20th percentile intensity value.

\section{Chemical and optical perturbations in BIO-133 based imaging devices}

\section{Fabrication of chemical delivery devices for cells}

A modified PDMS well plate design (Supplementary Fig. 11) was applied to deliver chemical perturbations to cells (Fig. 4a-c). A $400 \mu \mathrm{m}$ thick BIO-133 film was created using the method described above. $10 \mathrm{~mL}$ PDMS was cured in a $10 \mathrm{~cm}$ dish, PDMS tubing (Dow Corning, 508-004) was placed on the cured PDMS layer at $8 \mathrm{~mm}$ intervals, and another $15 \mathrm{~mL}$ PDMS was added to obtain a $\sim 4 \mathrm{~mm}$ thick PDMS slab with channels contained inside. Holes crossing the channels were punched at $8 \mathrm{~mm}$ intervals using a $5 \mathrm{~mm}$ diameter circular punch (Acuderm Inc., P550). A piece of double-sided silicone-based adhesive tape (Adhesives Research, ARCare 90880) was also punched at $8 \mathrm{~mm}$ intervals using a $2.5 \mathrm{~mm}$ diameter circular punch (Acuderm Inc., P2550). The PDMS slab and the tape were cut into two-well pieces. BIO-133 film, PDMS chunks and double-sided tape were disinfected in 75\% ethanol. After disinfection, the BIO-133 membrane was adhered to PDMS via the double-sided adhesive tape, so that the matching holes became wells for cell culture. After growing cells, tubing (Scientific Commodities Inc., BB31695-PE/2) was inserted into both sides of the channel for introducing chemical flow and another piece of double-sided tape without holes was used to seal the wells. The assembly was flipped over for diSPIM imaging through the BIO-133 membrane.

\section{Mitochondrial imaging in the presence of CCCP}


568 U2OS cells were cultured in two $5 \mathrm{~mm}$ diameter BIO-133 bottomed wells with ports and 569 transfected with 300-400 ng of mEmerald-Tomm20. Before imaging, the wells were filled with 570 live cell imaging solution (Invitrogen, A14291DJ), flipped over and attached to a $10 \mathrm{~cm}$ petri dish 571 with double-sided silicone-based adhesive tape (Adhesives Research, ARCare 90880). For the 572 well containing control cells, tubing was left disconnected from a source. For the well

573 containing cells that experienced chemical perturbation, input tubing was connected to a 574 syringe containing $0.05 \mathrm{mM}$ carbonyl cyanide m-chlorophenyl hydrazine (CCCP, Sigma, C2759).

575 The syringe is higher than the output tube so that drug flow was induced by gravity. We used a

576 valve (McMaster-Carr, 7033T21) placed between the input tube and syringe to control the flow.

577 The valve was closed prior to imaging. For each well, two cells were chosen for imaging. A

578 multi-position acquisition was set in the Micro-manager ${ }^{69}$ diSPIM plugin $^{70}$ to sequentially image

579 the four cells. Volume acquisition time was $3 \mathrm{~s}$, and 90 volumes were acquired for each cell with

58060 s intervals between volumes. 10 minutes after the imaging started, the valve was opened

581 and drug flow was induced in $\sim 60 \mathrm{~s}$. Dual-view images were deconvolved with ImageJ plugin

582 DiSPIM Fusion ${ }^{54}$, drift corrected (ImageJ plugin Correct 3D Drift,

583 https://imagej.net/Correct_3D_Drift) and bleach corrected (ImageJ function Bleach Correction, https://imagej.net/Bleach Correction, exponential fitting method).

\section{Encapsulation of C. elegans into PEG hydrogels} hydrogel precursor solutions were prepared by combining $20 \% \mathrm{w} / \mathrm{v}$ poly(ethylene glycol) diacrylate (PEG-DA, $3350 \mathrm{MW}, 94.45 \%$ acrylation, ESI BIO) with 0.10\% w/v Irgacure 2959 photoinitiator (2-hydroxy-4'-(2-hydroxyethoxy)-2-methylpropiophenone, 12959, BASF) in 1x Sbasal buffer ( $100 \mathrm{mM} \mathrm{NaCl}, 50 \mathrm{mM} \mathrm{KPO}_{4}$ buffer, $\mathrm{pH}$ 6.0). Clean $24 \mathrm{~mm} \times 50 \mathrm{~mm}$ glass coverslips (VWR) were rendered permanently hydrophobic by exposure to vapors of (tridecafluoro1,1,2,2-tetrahydrooctyl) trichlorosilane (Gelest). For covalent attachment of the PEG hydrogel to glass, coverslips (Thermo Scientific) were silanized by coating with 3-(trimethoxysilyl)propyl methacrylate (TMSPMA, Sigma-Aldrich). Both methods of surface modification were applied to 1 " $\times 3$ " glass slides (VWR). A small volume $(1.75 \mu \mathrm{L})$ of PEG hydrogel solution with photoinitiator was pipetted onto a hydrophobic glass slide flanked by two PDMS spacers whose thickness matched the desired hydrogel thickness of $150 \mu \mathrm{m}$. Animals were transferred into the hydrogel solution by worm pick. A coverslip, TMSPMA treated for making mounted PEG-DA gels, or untreated for making freestanding gels, was placed over the hydrogel droplet and supported by the PDMS spacers. The glass slide/coverslip sandwich was then placed over a UV light source (312 nm, International Biotechnologies, Inc, model UVH, 12W) and illuminated for two minutes until gelation. Hydrogel disks were immediately transferred to wet agar dishes to keep embedded animals hydrated. Corning) in a ratio of 1:10 and poured to a depth of $5 \mathrm{~mm}$ on a silicon master positive mold of the microchannels used previously ${ }^{71}$. Once cut free of the master, devices were punched so that two balanced-length inlets and the outlet had $1.5 \mathrm{~mm}$ holes going through the thickness of the material, and $1 \mathrm{~mm}$ holes punched from the side to allow flexible tubing to be inserted from 
612 the sides. The smooth PDMS device surface opposite the microchannels was irreversibly

613 bonded to a glass slide using oxygen plasma (Harrick PDC-32G, 18W, 45 seconds). A thin PDMS

614 membrane $(150 \mu \mathrm{m})$ was cut with a $3.5 \mathrm{~mm}$ diameter dermal punch and then oxygen plasma

615 bonded to the microfluidic channel surface with the hole in the membrane exposing the

616 micropost array. The hole in the thin PDMS membrane formed a "well" that hydrogel disks

617 could be gently placed in with forceps. A thin BIO-133 membrane (75-80 $\mu \mathrm{m})$ was prepared by

618 gelation of BIO-133 liquid polymer between two glass slides rendered permanently

619 hydrophobic as described above. Two layers of clear cellophane tape (height of 80 $\mu \mathrm{m}$ )

620 formed the standoffs that determined final membrane height. After degassing the microfluidic

621 device in a vacuum chamber for approximately 45 minutes, the device was removed from the

622 desiccator, connected to tubing, and flushed with S. basal buffer before use to remove any air

623 bubbles. Hydrogel disks could be interchanged between the well formed by the PDMS above

624 the micropost array easily using forceps and then the system sealed for microfluidic flow using

625 the thin BIO-133 membrane described above. See also Supplementary Fig. 12.

628 For both wide-field and diSPIM assays, diacetyl (2,3-butanedione, Sigma) was diluted to $1.1 \mu \mathrm{M}$

629 in $1 \times \mathrm{S}$. basal ( $10^{-7}$ dilution). $1 \mu \mathrm{L}$ of $1 \mathrm{mg} / \mathrm{mL}$ fluorescein solution was added to $40 \mathrm{~mL}$ of diacetyl solution to visualize stimulus delivery.

Wide-field Imaging with Chemical Stimulation

633 For wide-field, single plane imaging of multiple $C$. elegans at once ${ }^{57}$, the microfluidic chamber, valves, tubing and reservoirs were prepared as above and placed on a Zeiss AxioObserver epifluorescence microscope with a 5x, 0.25 NA objective, EGFP filter set, and Hamamatsu OrcaFlash 4 sCMOS camera. Micromanager scripts ${ }^{72}$ automatically synchronized capture of ten 30-s trials recording at $10 \mathrm{fps}$ with $10 \mathrm{~ms}$ excitation pulses and $10 \mathrm{~s}$ chemical stimulation. NeuroTracker software ${ }^{71}$ analyzed the wide-field neural imaging data, from which backgroundcorrected fluorescence changes were calculated in MATLAB as $\triangle F / F_{0}$, where $F_{0}$ is baseline neural fluorescence during the four seconds prior to stimulation. Data for multiple individual animals were also presented as a population mean to show the relative decrease in average calcium response after multiple stimulation periods.

\section{DiSPIM Imaging with Optical and Chemical Stimulation}

Stimulus control for optical illumination or chemical pulses was integrated with diSPIM volumetric imaging using a custom Micromanager script controlling an Arduino Uno and enabling independent digital switching of 6 TTL channels at the beginning of specified image acquisition timepoints. One TTL channel controlled the intensity of a red LED $(617 \mathrm{~nm}, 3 \mathrm{~W}$, Mightex) connected to the bottom port of the diSPIM and illuminated the sample through a Nikon 4x, 0.1 NA lower objective. A second TTL channel controlled a $12 \mathrm{~V}$ fluidic valve system for chemical stimulation (ValveLink 8.2, Automate). Pinch valves allowed flow of either buffer or chemical stimulus lines into the microfluidic channel network, flowing to a common outlet.

655

For optogenetic stimulation experiments, animals were embedded in BIO-133 disks bonded to a cover glass placed in the diSPIM sample chamber. To embed animals, they were first transiently 
656 immobilized by being picked onto seeded (OP50 E. coli) plates with $1 \mathrm{mM}$ tetramisole, and 657 allowed to rest for 1.5 hours. Subsequently, worms were picked into a droplet of BIO-133

658 polymer liquid and gelled in the same manner as the PEG-DA hydrogel disks above, using a 659 TMSPMA silanized coverslip for covalent bonding.

For chemical stimulation experiments, animals were embedded in PEG hydrogel disks. Animals can be maintained in these disks for many hours if they are kept hydrated ${ }^{7}$. Just prior to an experiment, an animal-embedded disk was inserted into the sample cavity of the diSPIM microfluidic chamber. A 75-80 $\mu \mathrm{m}$ thick BIO-133 membrane was sealed to the microfluidic device surface, closing the fluidic channel with the PEG disk and animals contained within. The hydrogel disk was inserted into a droplet of S. basal buffer present in the well to avoid the introduction of bubbles that would disrupt microfluidic flow. To assure continuous microfluidic 668 flow through the chamber without leaking, we balanced inlet and outlet flows by adjusting the reservoir heights. Specifically, inlet reservoir heights were held slightly above the stage $\left(\Delta h_{\text {in }}\right)$, and the outlet reservoir level was placed further below the stage $\left(\Delta h_{\text {out }}>\Delta h_{\text {in }}\right)$ to ensure a slight negative pressure in the chamber. Microfluidic stimulus switching was achieved using a dual pinch valve (NResearch Inc., 161P091), that alternately allows either a buffer or stimulus line to flow through the microfluidic chamber to the single outflow line.

A typical diSPIM acquisition captured one volume per second (10 ms exposure, minimum slice time setting, 55 slices per volume, $166.4 \times 166.4 \times 82.5 \mu \mathrm{m}$ total volume space) for 10 minutes, with 20-s duration stimulation every minute. Z-Projection time series videos were produced in Image from cropped versions of the total number of images, then analyzed for GCaMP neural 679 fluorescence using rectangular boxes for integrated fluorescence density, with a nearby region void of signal used for background subtraction. 
a

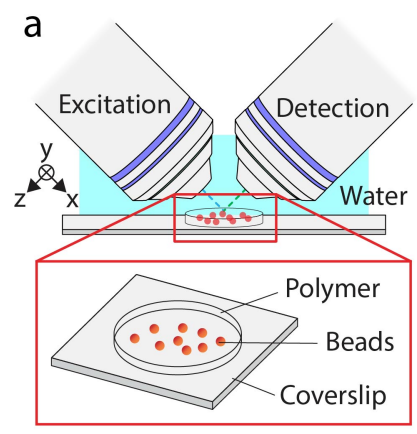

b

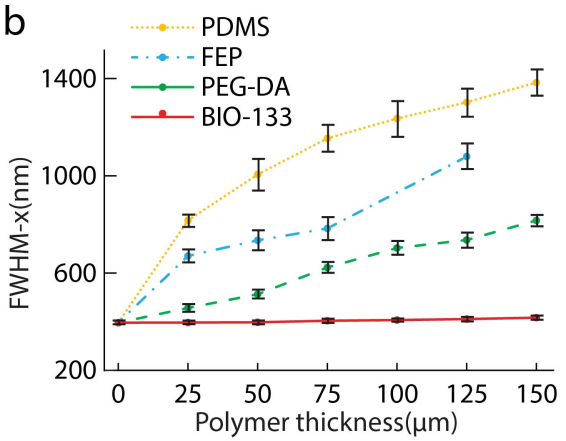

C Water BIO-133 PEG-DA FEP PDMS $\mathrm{n}=1.333 \mathrm{n}=1.333 \mathrm{n}=1.345 \mathrm{n}=1.344 \mathrm{n}=1.407$

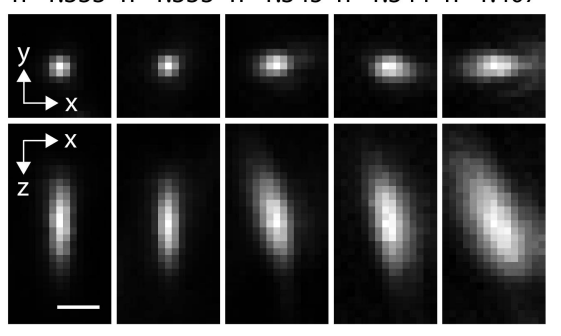

Polymer thickness: $75 \mu \mathrm{m}$

Fig. 1, Diffraction-limited imaging is possible when imaging through BIO-133, unlike other polymers. a) Imaging geometry. A light sheet is used to illuminate the $100 \mathrm{~nm}$ yellow-green bead sample, which is embedded under progressively thicker polymer. Illumination and detection occur through 0.8 NA water-dipping objectives. b) Full width at half maximum (FWHM) in the ' $x$ ' direction under different thicknesses of polymer. Means and standard deviations are shown. c) Exemplary lateral (top row) and axial (bottom row) images of beads imaged through $75 \mu \mathrm{m}$ of polymer, demonstrating that BIO-133 provides diffraction-limited performance whilst the other polymers do not. Single images, rather than maximum intensity projections, are shown. The refractive index of each polymer as measured with a refractometer is also indicated (average value from 3 independent trials). Scale bar: $1 \mu \mathrm{m}$. See also 
698

699

700

701

702

703

704

705

706

707

708

709

710

711

712

713

714

715

716

717

718

719

720

721

722

723

724
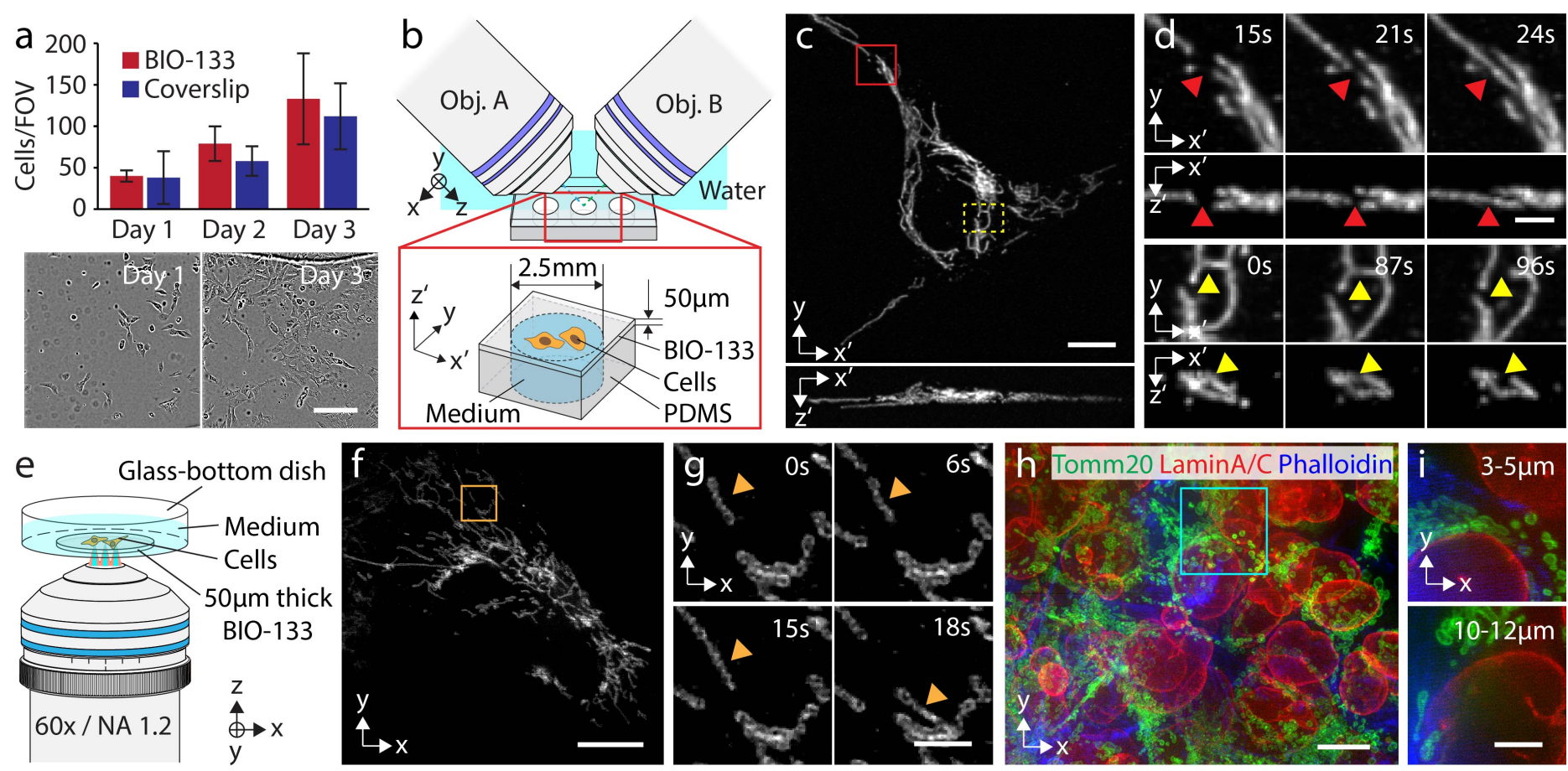

Fig. 2, BIO-133 provides an inert and biocompatible scaffold on which to grow and image cells. a) U2OS growth on $\mathrm{BIO}-133$ is similar to growth on glass coverslips. Top: quantifying cell growth on $50 \mu \mathrm{m}$ thick BIO-133 layer vs. glass coverslip. Means and standard deviations from 3 fields of view (10x magnification, $\sim 800 \mu \mathrm{m} \times 800 \mu \mathrm{m}$ field of view) are shown over 3 days. Bottom: example fields of view from day 1 and day 3, cells on BIO-133 layer. Scale bar: $200 \mu \mathrm{m}$. See also Supplementary Fig. 2, 3. b) Schematic of diSPIM imaging geometry. $50 \mu \mathrm{m}$ film with adherent cells is inverted and imaged in the diSPIM setup. See also Supplementary Fig. 4. c) Example maximum intensity projections of deconvolved images of U2OS cells expressing mEmerald-Tomm20 in lateral (top) and axial (bottom) views. Scale bar: $10 \mu \mathrm{m}$. d). Higher magnification views of the red and yellow rectangles in c), highlighting examples of mitochondrial fusion (top, red arrowhead) and fission (bottom, yellow arrowhead). 50 volumes were taken with a 3 s inter-volume interval. See also Supplementary Video 1 . Note that primed coordinates refer to the plane of the BIO-133 layer $\left(x^{\prime}, y\right)$ and the direction normal to the BIO133 layer ( $\left.z^{\prime}\right)$. Scale bar: $2 \mu \mathrm{m}$. e) iSIM imaging geometry. Cells were cultured on $50 \mu \mathrm{m}$ BIO-133 film, and the film placed in a glass-bottom dish and immersed in cell culture medium. Imaging was performed with a 60x, NA 1.2 water-immersion lens. f) Example deconvolved iSIM maximum intensity projection showing live U2OS cells expressing mEmerald-Tomm20. Scale bar: $10 \mu \mathrm{m}$. g) Higher magnification view of orange rectangular region in f). Orange arrowhead marks the same mitochondrion. 25 volumes were acquired with a $3 \mathrm{~s}$ inter-volume interval. See also Supplementary Videos, 3, 4. Scale bar: $2 \mu \mathrm{m}$. A 0.5 pixel median filter was used to denoise images in $\mathbf{f}, \mathbf{g}$ ) prior to display. $\mathbf{h}$ ) Multiple layers of HCT-116 cells were grown on $50 \mu \mathrm{m} \mathrm{BIO-}$ 133 layer and immunostained against Tomm20 (green), lamin A/C (red), and actin (blue). See also Supplementary Video 5. Scale bar: $10 \mu \mathrm{m}$. i) Maximum intensity projection over indicated axial range (measured from the bottom of the cell layer) for cyan rectangular region in $\mathbf{h}$ ). Scale bar: $5 \mu \mathrm{m}$. 


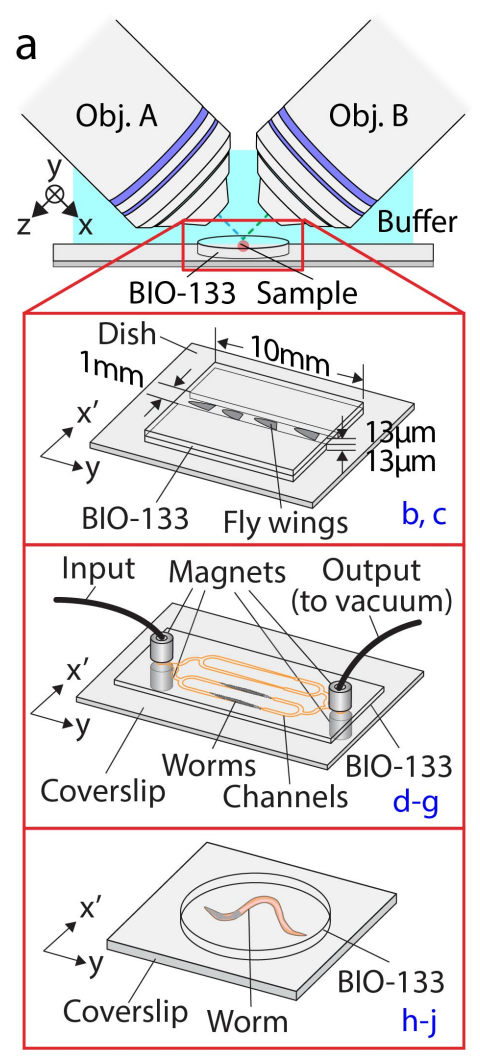

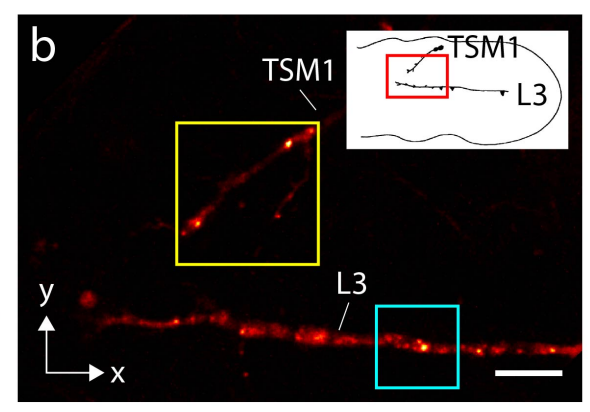
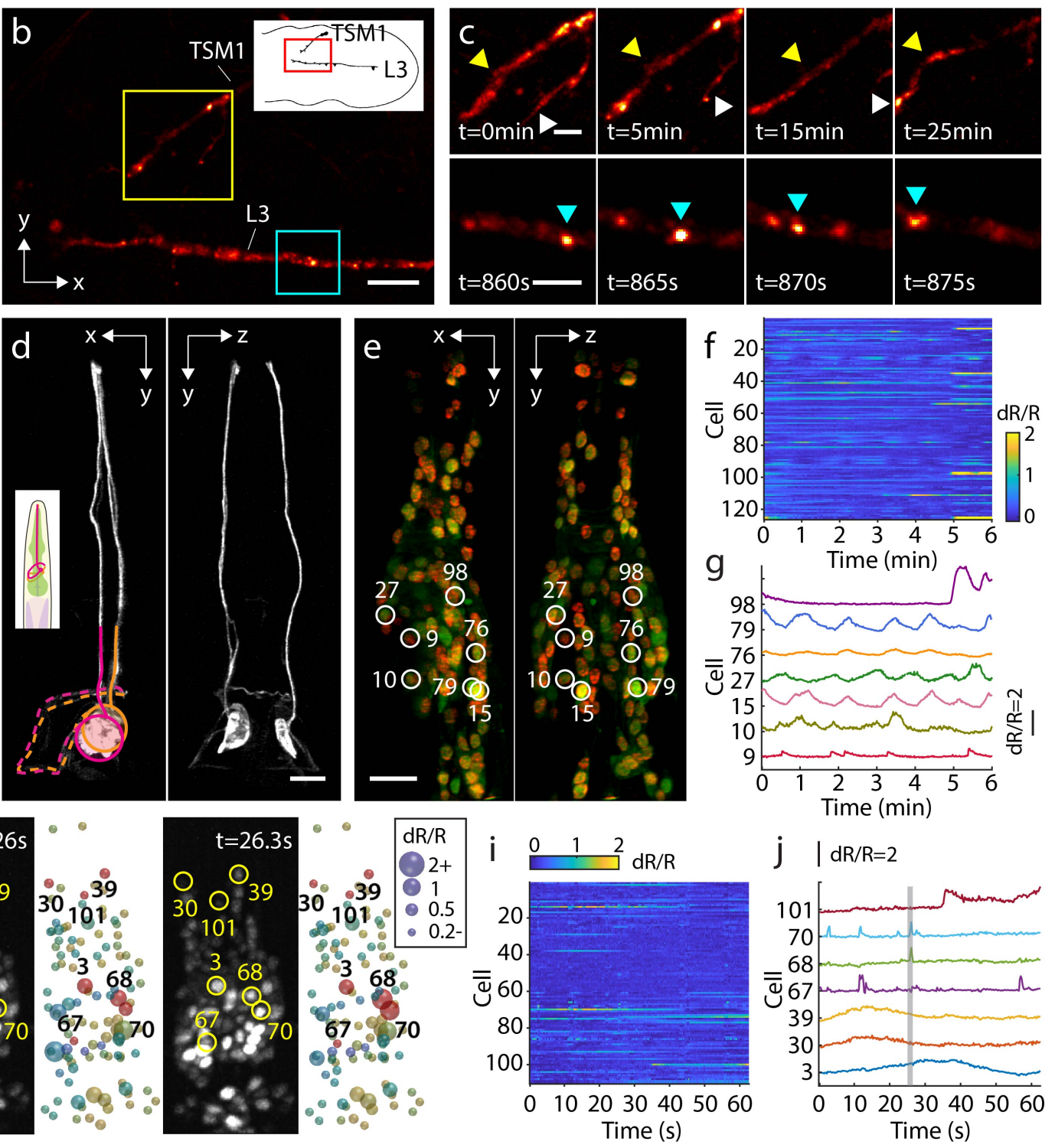

Fig. 3 Live imaging of BIO-133 encapsulated fly wings and C. elegans. a) Experimental

schematic. Top: A thin layer of BIO-133 membrane covers excised fly wings, immobilizing them so that axon dynamics can be recorded at high resolution over an extended period. Middle: A simple microfluidic device is used to trap adult worms for structural and functional imaging of the nervous system. Bottom: Worms can also be encapsulated in a gelled droplet of BIO-133. See also Supplementary Figs. 8, 10. b) Deconvolved, single-view maximum intensity projection of a fly wing with TdTomato-labelled CD4 showing the axons of two neurons (upper: TSM1; lower: L3) shortly before fasciculation in the developing Drosophila melanogaster wing disc. Scale bar: $10 \mu \mathrm{m}$. 360 volumes were taken with $5 \mathrm{~s}$ inter-volume intervals. (30 min in total, see also Supplementary Video 6). c) Magnified regions of TSM1 and L3 axons, corresponding to yellow and blue rectangles in b), highlighting morphological changes and apparent motion of CD4 puncta. Scale bars: $4 \mu \mathrm{m}$. d) Isotropic, high-resolution imaging of GFP-labeled axons and dendrites in anesthetized adult $C$. elegans, as shown by orthogonal, jointly deconvolved diSPIM maximum intensity projections. Cell bodies are circled and axons entering the nerve ring region 
740 are overlaid with dotted lines. Scale bar: $10 \mu \mathrm{m}$. e) Calcium imaging of adult worm (red channel:

741 TagRFP, green channel: GCaMP6s; both labels targeted to nuclei), 2 views imaged at $1.25 \mathrm{~Hz}$

742 volumetric rate. Joint deconvolution diSPIM results are shown; red and green channels were

743 simultaneously collected and colors are overlaid in display. Scalebar: $10 \mu \mathrm{m}$. See also

744 Supplementary Video 7. f) dR/R traces for all 126 tracked nuclei. g) dR/R traces for selected

745 individual neurons. Note correspondence with numbered neurons and marked neurons in e). h)

746 Calcium imaging of larval worm with higher temporal resolution (4 Hz volumetric rate), single-

747 view results are shown. GCaMP channel and associated segmented $\mathrm{dR} / \mathrm{R}$ signal are indicated for

7483 successive time points. Scale bar: $5 \mu \mathrm{m}$. See also Supplementary Video 8. i) dR/R traces for all

749110 nuclei segmented and tracked in h). j) traces for selected individual neurons. Note

750 correspondence with numbered neurons and marked neurons in $\mathbf{h}$ ). 

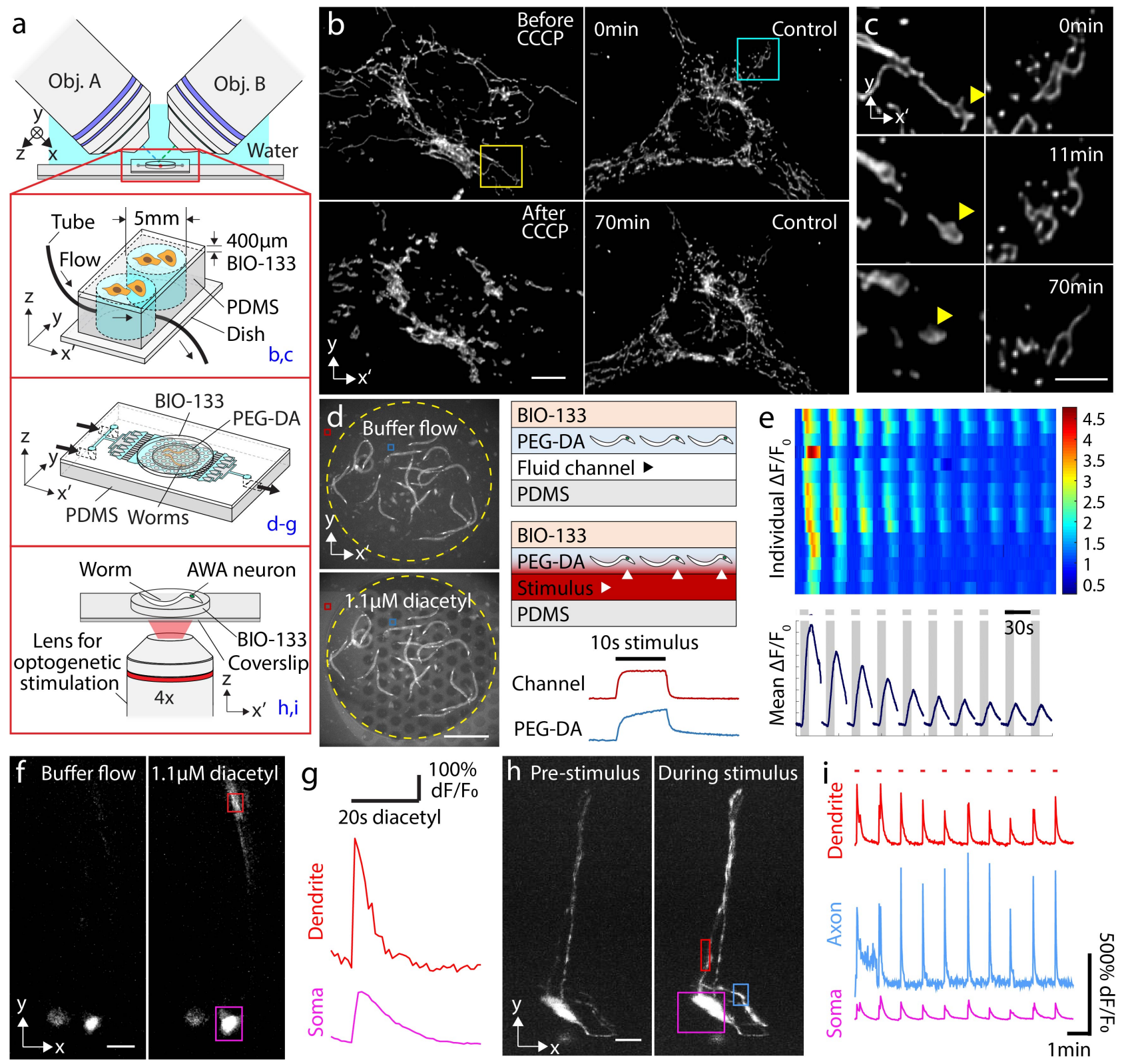

Fig. 4, BIO-133 is compatible with chemical and optical perturbations. a) Experimental schematic for perturbations. Cells grown on BIO-133 were placed on PDMS wells and were either perturbed by flowing $0.05 \mathrm{mM}$ CCCP or left as controls (top higher magnification view). Alternatively, worms were embedded in PEG-DA bonded to a PDMS flow chip and imaged through a layer of BIO-133 (middle higher magnification view) to examine response to chemical stimulation; or embedded in BIO-133, repetitively stimulated with red light from lower, $4 x$ objective and imaged using upper diSPIM objectives (bottom higher magnification view). See also Supplementary Figs. 11-13. b) Example cells with (left column) and without (right column) CCCP treatment at early (top, $0 \mathrm{~min}$ ) and late (bottom, $70 \mathrm{~min}$ ) time points. CCCP was added at 10 minutes. Maximum intensity projections of deconvolved diSPIM data are shown. Scale bar:

$76410 \mu \mathrm{m}$. See also Supplementary Video 9. c) Higher magnification views of yellow (left column) 765 and blue (right column) regions in b). Yellow arrowhead shows CCCP-induced morphological 
change of mitochondrion. Scale bar: $5 \mu \mathrm{m}$. d) Example images of worms expressing GCaMP immobilized in PEG-DA disk with (bottom) and without (top) $1.1 \mu \mathrm{M}$ diacetyl. Fluorescein added to stimulus highlights the rapid addition/removal of chemical. Scale bar: $500 \mu \mathrm{m}$. Right schematics show layered structure of assembly, including direction of flow and diffusion (arrowheads) into PEG-DA layer. Line plots show intensity of fluorescein over time in channel (red) and PEG-DA (blue) layers. e) Top: dF/F heatmaps derived from widefield microscopy measurements from 15 animals (rows) in response to 10 repeated stimulus pulses (once per minute). Bottom: responses averaged over all animals show neural adaptation. f) Single-view diSPIM images recorded from a single animal, showing subcellular response in AWA neuron to $1.1 \mu \mathrm{M}$ diacetyl compared to control (buffer flow) conditions. Contrast has been adjusted to better highlight the response from different cell regions. Scale bar: $10 \mu \mathrm{m}$. See also

777 Supplementary Video 10. g) Graphs show average intensity from boxed regions in f)

778 highlighting fluorescence intensity changes in soma and dendrite. $\mathbf{h}$ ) Worms expressing 779 Chrimson and GCaMP are repetitively stimulated with red light and imaged using upper diSPIM objectives. Maximum intensity projection of GCaMP fluorescence from single-view diSPIM recordings are shown before (left) and after (right) optogenetic stimulation. Scale bar: $10 \mu \mathrm{m}$.

782 See also Supplementary Video 11. i) dF/F traces for dendrite, axon, and soma, corresponding to 783 boxed regions in $\mathbf{h})$. 


\section{References}

7861 Chalfie, M., Tu, Y., Euskirchen, G., Ward, W. W. \& Prasher, D. C. Green fluorescent

787 protein as a marker for gene expression. Science 263, 802-805 (1994).

7882 Ou, G., Stuurman, N., D'Ambrosio, M. \& Vale, R. D. Polarized Myosin Produces Unequal-

789 Size Daughters During Asymmetric Cell Division. Science 330, 677-680 (2010).

7903 Kato, S. et al. Global brain dynamics embed the motor command sequence of

$791 \quad$ Caenorhabditis elegans. Cell 163, 656-669 (2015).

7924 Chokshi, T. V., Ben-Yakar, A. \& Chronis, N. CO2 and compressive immobilization of C.

$793 \quad$ elegans on-chip. Lab Chip 9, 151-157 (2009).

7945 Snow, J. J. et al. Two anterograde intraflagellar transport motors cooperate to build

795 sensory cilia on C. elegans neurons. Nature Cell Biology 6, 1109-1113 (2004).

7966 Kim, E., Sun, L., Gabel, C. V. \& Fang-Yen, C. Long-Term Imaging of Caenorhabditis

797 elegans Using Nanoparticle-Mediated Immobilization. PLoS One, e53419 (2013).

7987 Burnett, K., Edsinger, E. \& Albrecht, D. R. Rapid and gentle hydrogel encapsulation of

799 living organisms enables long-term microscopy over multiple hours. Communications

800 biology 1, 73 (2018).

8018 Rohde, C. B., Zeng, F., Gonzalez-Rubio, R., Angel, M. \& Yanik, M. F. Microfluidic system

802

803

804 for on-chip high-throughput whole-animal sorting and screening at subcellular resolution. Proc Natl Acad Sci U S A 104, 13891-13895 (2007).

805

806 Cornaglia, M., Lehnert, T. \& Gijs, M. A. M. Microfluidic systems for high-throughput and high-content screening using the nematode Caenorhabditis elegans. Lab Chip 17, 37363759 (2017).

11 Mondal, S. et al. Large-scale microfluidics providing high-resolution and high-throughput screening of Caenorhabditis elegans poly-glutamine aggregation model. Nat Commun. 7, 13023 (2016).

12 Levario, T. J., Zhan, M., Lim, B., Shvartsman, S. Y. \& Lu, H. Microfluidic trap array for massively parallel imaging of Drosophila embryos. Nat Protoc. 8, 721-736 (2013).

13 Yanik, M. F., Rohde, C. B. \& Pardo-Martin, C. Technologies for micromanipulating, imaging, and phenotyping small invertebrates and vertebrates. Annu Rev Biomed Eng 13, 185-217 (2011).

14 Shorr, A. Z., Sönmez, U. M., Minden, J. S. \& LeDuc, P. R. High-throughput

82015 Stavrakis, S., Holzner, G., Choo, J. \& DeMello, A. High-throughput microfluidic imaging mechanotransduction in Drosophila embryos with mesofluidics Lab Chip 19, 1141-1152 and 'real-time' cell classification using convolutional neural networks for higher efficiency in single-cell cloning. Scientific Reports 10, 1193 (2020). 
82818 Anagnostidis, V. et al. Deep learning guided image-based droplet sorting for on-demand selection and analysis of single cells and 3D cell cultures Lab Chip 20, 889-900 (2020).

83019 Lam, J. et al. Adaptation of a Simple Microfluidic Platform for High-Dimensional Quantitative Morphological Analysis of Human Mesenchymal Stromal Cells on Polystyrene-Based Substrates. SLAS Technol. 22, 646-661 (2017). Doolin, M. T. \& Stroka, K. M. Integration of Mesenchymal Stem Cells into a Novel Micropillar Confinement Assay. Tissue Engineering Part C: Methods 25, 662-676 (2019). Albrecht, D. R. et al. Microfluidics-integrated time-lapse imaging for analysis of cellular dynamics. Integrative Biology 2, 278-287 (2010).

22 Reilly, D. K., Lawler, D. E., Albrecht, D. R. \& Srinivasan, J. Using an Adapted Microfluidic Olfactory Chip for the Imaging of Neuronal Activity in Response to Pheromones in Male C. Elegans Head Neurons. JoVE 127, e56026 (2017). Yemini, E. et al. NeuroPAL: A Neuronal Polychromatic Atlas of Landmarks for WholeBrain Imaging in C. elegans. bioRxiv, https://doi.org/10.1101/676312 (2019). Geometry at Microscale for High-Throughput Visual Screens in Neurodegeneration and Neuronal Development Studies. PLoS One 7, e35037 (2012).

26 Belfer, S. J. et al. Caenorhabditis-in-Drop Array for Monitoring C. elegans Quiescent Behavior. Sleep 36, 689-698 (2013). automatically measuring pharyngeal pumping in C. elegans. Journal of Neuroscience Methods 274, 172-178 (2016). high-content phenotyping from the first larval stage till the onset of adulthood of the nematode Caenorhabditis elegans. Lab Chip 19, 120-135 (2018).

29 Hulme, S. E. et al. Lifespan-on-a-chip: microfluidic chambers for performing lifelong observations of C. elegans. Lab Chip 10, 589-597 (2010). Hwang, H., Krajniak, J., Matsunaga, Y., Benian, G. M. \& Lu, H. On-demand optical immobilization of Caenorhabditis elegans for high-resolution imaging and microinjection. Lab Chip 14, 3498-3501 (2014).

31 Krajniak, J. \& Lu, H. Long-term high-resolution imaging and culture of C. elegans in chipgel hybrid microfluidic device for developmental studies. Lab Chip 10, 1862-1868 (2010). S., N., Stowers, R., Lou, J., Xia, Y. \& Chaudhuri, O. Varying PEG density to control stress relaxation in alginate-PEG hydrogels for 3D cell culture studies Biomaterials 200, 15-24 (2019).

33 Zhang, Z. F., Ma, X., Wang, H. \& Ye, F. Influence of polymerization conditions on the refractive index of poly(ethylene glycol) diacrylate (PEGDA) hydrogels. Applied Physics $A$ 124, 283 (2018).

34 Wevers, N. R. et al. High-throughput compound evaluation on 3D networks of neurons and glia in a microfluidic platform. Scientific Reports 6, 38856 (2016). 
87135 Pittman, W. E., Sinha, D. B., Zhang, W. B., Kinser, H. E. \& Pincus, Z. A simple culture system for long-term imaging of individual C. elegans. Lab Chip 17, 3909-3920 (2017).

87336 Kim, D. N. H., Kim, K. T., Kim, C., Teitell, M. A. \& Zangle, T. A. Soft lithography fabrication of index-matched microfluidic devices for reducing artifacts in fluorescence and quantitative phase imaging. Microfluidics and Nanofluidics 22 (2017). Polanco, E. R., Western, N. \& Zangle, T. A. Fabrication of Refractive-index-matched Devices for Biomedical Microfluidics. J Vis Exp 139, 58296 (2018).

Levario, T. J., Insley, P., Hwang, H., Shaham, S. \& Lu, H. in 18th International Conference on Miniaturized Systems for Chemistry and Life Sciences, MicroTAS 2014 727-729 (San Antonio, TX, 2014).

$39 \mathrm{Xu}, \mathrm{T}$. et al. Modified inverted selective plane illumination microscopy for submicrometer imaging resolution in polydimethylsiloxane soft lithography devices. Lab Chip Advance Online Publication, doi:https://doi.org/10.1039/D0LC00598C (2020). cellular-resolution vertebrate screening platform with parallel animal processing. $L a b$ Chip 12, $711-716$ (2012). resolution imaging and application to sub-wavelength particle trapping. Lab Chip 16, 465-470 (2016). planar illumination microscopy. Optics Letters 33, 2302-2304 (2008). Wu, Y. et al. Spatially isotropic four-dimensional imaging with dual-view plane illumination microscopy. Nat Biotechnol. 31, 1032-1038 (2013).

894

44 Chen, B. C. et al. Lattice light-sheet microscopy: imaging molecules to embryos at high spatiotemporal resolution. Science 346, 1257998 (2014). Hedde, P. N., Malacrida, L., Ahrar, S., Siryaporn, A. \& Gratton, E. sideSPIM - selective plane illumination based on a conventional inverted microscope. Biomedical Optics Express 8, 3918-3937 (2017). imaging. Nature Protocols 9, 2555-2573 (2014).

47 Kaufmann, A., Mickoleit, M., Weber, M. \& Huisken, J. Multilayer mounting enables longterm imaging of zebrafish development in a light sheet microscope. Development 139, 3242-3247 (2012).

905

48 York, A. G. et al. Instant super-resolution imaging in live cells and embryos via analog image processing. Nat Methods 10, 1122-1126 (2013). Developing Wing of Drosophila melanogaster. Developmental Biology 104, 259-273 (1984).

50 Jan, Y. N., Ghysen, A., Christoph, I., Barbel, S. \& Jan, L. Y. Formation of Neuronal Pathways in the Imaginal Discs of Drosophila melanogaster. The Journal of Neuroscience 5, 2453-2464 (1985).

51 Clarke, A. et al. Dynamic Morphogenesis of a Pioneer Axon in Drosophila and Its Regulation by Abl Tyrosine Kinase. Mol Biol Cell 31, 452-465 (2020). 
91452 Clarke, A. et al. Abl Signaling Directs Growth of a Pioneer Axon in Drosophila by Shaping

915

916

917

918

919

920

921

922

923

924

925

926

927

928

929

930

931

932

933

934

935

936

937

938

939

940

941

942

943

944

945

946

947

948

949

950

951

952

$953 \quad 69$

954

955

956

the Intrinsic Fluctuations of Actin. Mol Biol Cell 31, 466-477 (2020).

53 Hulme, S. E., Shevkoplyas, S. S., Apfeld, J., Fontana, W. \& Whitesides, G. A

Microfabricated Array of Clamps for Immobilizing and Imaging C. Elegans. Lab Chip 7, 1515-1523 (2007).

54 Guo, M. et al. Rapid image deconvolution and multiview fusion for optical microscopy. Nature Biotechnol., in press, doi:https://doi.org/10.1038/s41587-020-0560-x (2020).

55 Chen, T.-W. et al. Ultra-sensitive fluorescent proteins for imaging neuronal activity. Nature 499, 295-300 (2013).

56 Nguyen, J. P., Linder, A. N., Plummer, G. S., Shaevitz, J. W. \& Leifer, A. M. Automatically tracking neurons in a moving and deforming brain. PLoS Comput Biol. 13, e1005517 (2017).

57 Larsch, J., Ventimiglia, D., Bargmann, C. I. \& Albrecht, D. R. High-throughput imaging of neuronal activity in Caenorhabditis elegans. PNAS 110, E4266-4273 (2013).

58 Sengupta, P., Chou, J. H. \& Bargmann, C. I. odr-10 Encodes a Seven Transmembrane Domain Olfactory Receptor Required for Responses to the Odorant Diacetyl. Cell 84, 899-909 (1996).

59 Shidara, H., Hotta, K. \& Oka, K. Compartmentalized cGMP Responses of Olfactory Sensory Neurons in Caenorhabditis elegans. Journal of Neuroscience 37, 3753-3763 (2017).

60 Klapoetke, N. C. et al. Independent optical excitation of distinct neural populations. Nature Methods 11, 338-346 (2014).

61 Martin, C. et al. Line excitation array detection fluorescence microscopy at 0.8 million frames per second Nature Communications 9, 4499 (2018).

62 Han, Y. et al. Cameraless high-throughput three-dimensional imaging flow cytometry. Optica 6, 1297-1304 (2019).

63 Ravasio, A., Vaishnavi, S., Ladoux, B. \& Viasnoff, V. High-resolution imaging of cellular processes across textured surfaces using an index-matched elastomer. Acta Biomater. 14, 53-60 (2015).

64 Xiao, F., Wen, X., Tan, X. H. M. \& Chiou, P.-Y. Plasmonic micropillars for precision cell force measurements across a large field-of-view Appl. Phys. Lett. 112, 033701 (2018).

65 Duffy, D. C., McDonald, J. C., Schueller, O. J. \& Whitesides, G. M. Rapid Prototyping of Microfluidic Systems in Poly(dimethylsiloxane). Anal Chem 70, 4974-4984 (1998).

66 Guo, M. et al. Single-shot super-resolution total internal reflection fluorescence microscopy. Nature Methods 15, 425-428 (2018).

67 Xia, Y. \& Whitesides, G. M. Soft Lithography. Angew. Chem. Int. Ed. Engl. 37, 550-575 (1998).

68 Atencia, J. et al. Magnetic connectors for microfluidic applications Lab Chip 10, 246-249 (2010).

69 Edelstein, A. D. et al. Advanced methods of microscope control using $\mu$ Manager software. Journal of Biological Methods 1, e11 (2014).

70 Ardiel, E. L. et al. Visualizing Calcium Flux in Freely Moving Nematode Embryos. Biophys. J. 112, 1975-1983 (2017). 
bioRxiv preprint doi: https://doi org/10.1101/2020 10.04.324996; this version posted October 11,2020 . The copyright holder for this preprint

(which was not certified by peer review) is the author/funder. This article is a US Government work. It is not subject to copyright under 17 USC 105 and is also made available for use under a CCO license.

95771 Lagoy, R. C. \& Albrecht, D. R. Microfluidic Devices for Behavioral Analysis, Microscopy, and Neuronal Imaging in Caenorhabditis elegans. Methods Mol Biol. 1327, 159-179

959 (2015).

$96072 \quad$ Lagoy, R. C. \& Albrecht, D. R. Automated fluid delivery from multiwell plates to microfluidic devices for high-throughput experiments and microscopy. Sci. Reports 8, 6217 (2018). 